Tropical Journal of Pharmaceutical Research July 2016; 15 (7): 1525-1533

ISSN: $1596-5996$ (print); 1596-9827 (electronic)

(C) Pharmacotherapy Group, Faculty of Pharmacy, University of Benin, Benin City, 300001 Nigeria.

All rights reserved.

Available online at http://www.tjpr.org

Original Research Article

http://dx.doi.org/10.4314/tjpr.v15i7.24

\title{
S-Alkylated/aralkylated 2-(1H-indol-3-yl-methyl)-1,3,4- oxadiazole-5-thiol derivatives. 2. Anti-bacterial, enzyme- inhibitory and hemolytic activities
}

\author{
Kaniz Rubab ${ }^{1}$, Muhammad A Abbasi ${ }^{1 *}$, Aziz-ur-Rehman ${ }^{1}$, Sabahat Z Siddiqui ${ }^{1}$, \\ Muhammad Ashraf ${ }^{2}$, Ayesha Shaukat ${ }^{2}$, Irshad Ahmad $^{3}$, Sidra Hassan ${ }^{3}$, \\ Muhammad A Lodhi ${ }^{4}$, Mehreen Ghufran ${ }^{4}$, Muhammad Shahid ${ }^{5}$ and Hina Fatima ${ }^{5}$ \\ ${ }^{1}$ Department of Chemistry, Government College University, Lahore-54000, ${ }^{2}$ Department of Biochemistry and Biotechnology, \\ ${ }^{3}$ Department of Pharmacy, The Islamia University of Bahawalpur, Bahawalpur-63100, ${ }^{4}$ Department of Biochemistry, Abdul Wali \\ Khan University, Mardan-23200, ${ }^{5}$ Department of Chemistry and Biochemistry, University of Agriculture, Faisalabad-38040, \\ Pakistan
}

*For correspondence: Email: atrabbasi@yahoo.com; abbasi@gcu.edu.pk; Tel: +92-42-111000010 ext 266

Received:

Revised accepted: 24 April 2016

\begin{abstract}
Purpose: To evaluate the antibacterial, enzyme-inhibitory and hemolytic activities of Salkylated/aralkylated 2-(1H-indol-3-ylmethyl)-1,3,4-oxadiazole-5-thiol derivatives.

Methods: Antibacterial activities of the compounds were evaluated using broth dilution method in 96 well plates. Enzyme inhibitory activities assays were investigated against a-glucosidase, butyrylcholinesterase (BchE) and lipoxygenase (LOX) using acarbose, eserine and baicalien as reference standards, respectively. A mixture of enzyme, test compound and the substrate was incubated and variation in absorbance noted before and after incubation. In tests for hemolytic activities, the compounds were incubated with red blood cells and variations in absorbance were used as indices their hemolytic activities.

Results: The compounds were potent antibacterial agents. Five of them exhibited very good antibacterial potential similar to ciprofloxacin, and had minimum inhibitory concentrations (MIC) of at least $9.00 \pm 4.12 \mu \mathrm{M}$ against S. aureus, E.coli, and B. subtilis. One of the compounds had strong enzyme inhibitory potential against $\alpha$-glucosidase, with $I C_{50}$ of $17.11 \pm 0.02 \mu \mathrm{g} / \mathrm{mL}$ which was better than that of standard acarbose ( $I C_{50} 38.25 \pm 0.12 \mu \mathrm{g} / \mathrm{mL}$ ). Another compound had $1.5 \%$ hemolytic activity.

Conclusion: S-Alkylated/aralkylated 2-(1H-indol-3-ylmethyl)-1,3,4-oxadiazole-5-thiol deviratives with valuable antibacterial, anti-enzymatic and hemolytic activities have been successfully synthesized. These compounds may be useful in the development of pharmaceutical products.
\end{abstract}

Keywords: 2-(1H-Indol-3-ylmethyl)-1,3,4-oxadiazole-5-thiol derivatives, Enzyme inhibition, Antibacterial activity, Hemolytic activity, Molecular docking

Tropical Journal of Pharmaceutical Research is indexed by Science Citation Index (SciSearch), Scopus, International Pharmaceutical Abstract, Chemical Abstracts, Embase, Index Copernicus, EBSCO, African Index Medicus, JournalSeek, Journal Citation Reports/Science Edition, Directory of Open Access Journals (DOAJ), African Journal Online, Bioline International, Open-J-Gate and Pharmacy Abstracts

\section{INTRODUCTION}

The synthesis and evaluation of 2, 5disubstituted-1,3,4-oxadiazole-2-thiol derivatives have received attention in the past decades. The medicinal values of these compounds are diverse, and include anti-edema and antiinflammatory, anti-microbial, analgesic, antimycobacterial, anti-convulsant, anti-tumor, antimalarial, anti-hepatitis B, anti-tuberculosis, insecticidal, anti-HIV and anti-Parkinsonism activities [1,2]. The ability of these compounds to 
inhibit certain enzymes such as cholinesterases, a-glucosidase and lipoxygenase can exploited for medicinal purposes. Cholinesterases are present in cholinergic and non-cholinergic tissues, as well as plasma and other body fluids [3-5]. a-glucosidase are used as oral antidiabetic drugs for the management of type-2 diabetes mellitus [6]. Lipoxygenase are important enzymes in the biosynthesis of leukotrienes used for the treatment of inflammation and allergic diseases $[7,8]$.

In continuation of our ongoing research efforts in search for new anti-bacterial, hemolytic and antienzymatic agents $[9,10]$, we report herein the pharmacological activities of Salkylated/aralkylated $\quad 2$-[1 $\mathrm{H}$-indol-3-ylmethyl]1,3,4-oxadiazole-5-thiols (6a-u)

\section{EXPERIMENTAL}

\section{Materials}

Three Gram negative bacterial strains (Escherichia coli, Pseudomonas aeruginosa and Salmonella typhi) and two Gram positive bacteria strains (Bacillus subtillis and Staphylococcus aureus) were clinically isolated and stored in agar culture medium for antibacterial evaluation. The enzymes, $\alpha$-glucosidase ( $\alpha$-D-glucoside glucohydrolase EC 3.2.1.20), butyrylcholinesterase (BChE, EC 3.1.1.8) and lipoxygenase (LOX, EC 1.13.11.12) were obtained from freshly heparinized human blood which was collected from volunteers.

\section{Lipoxygenase (LOX) assay}

Lipoxygenase activity was assayed according to standard methods [11-13]. The assay mixture $(200 \mu \mathrm{L})$ contained $150 \mu \mathrm{L}$ of sodium phosphate buffer (100 mM, pH 8.0), $10 \mu \mathrm{L}$ of test compound and $15 \mu \mathrm{L}$ of purified lipoxygenase. The mixture was pre-read at $234 \mathrm{~nm}$ and pre-incubated for 10 minutes at $25^{\circ} \mathrm{C}$. The reaction was then initiated by addition of $25 \mu \mathrm{L}$ of substrate solution. Change in absorbance was observed after 6 min at $234 \mathrm{~nm}$. All reactions were done in triplicates. Positive and negative controls were included in the assay. Quercetin $\left(0.5 \mathrm{mM}\right.$ well $\left.{ }^{-1}\right)$ was used as positive control. Inhibition (\%) was calculated as in Eq 1.

Inhibition $(\%)=\{(\mathrm{C}-\mathrm{T}) / \mathrm{C}\} 100$

where $C$ (i.e., control) = total enzyme activity without inhibitor, and $\mathrm{T}$ (i.e., test sample) = activity in the presence of test compound.
$\mathrm{IC}_{50}$ values (concentration that results in $50 \%$ enzyme inhibition) of the derivatives were calculated using EZ-Fit Enzyme kinetics software (Perella Scientific Inc. Amherst, USA).

\section{a-Glucosidase inhibitory assay}

a-Glucosidase inhibitory activity was determined according to the method of Pierre [14]. The assay mixture $(100 \mu \mathrm{L})$ contained $70 \mu \mathrm{L}$ of 50 $\mathrm{mM}$ phosphate buffered saline $(\mathrm{pH} \mathrm{6.8);10 \mu l}$ $(0.5 \mathrm{mM})$ of test compound and $10 \mu \mathrm{l}(0.057$ units) enzyme. The contents were mixed, preincubated for $10 \mathrm{~min}$ at $37{ }^{\circ} \mathrm{C}$ and pre-read at $400 \mathrm{~nm}$. Then $10 \mu \mathrm{l}$ of $0.5 \mathrm{mM}$ substrate ( $\mathrm{p}-$ nitrophenyl glucopyranoside) was added to initiate the reaction. Acarbose was used as positive control. After $30 \mathrm{~min}$ of incubation at 37 ${ }^{\circ} \mathrm{C}$, absorbance was measured at $400 \mathrm{~nm}$ using Synergy HT microplate reader. All experiments were carried out in triplicate. Inhibition (\%) was calculated as in Eq 2.

Inhibition $(\%)=\{(\mathrm{C}-\mathrm{T}) / \mathrm{C}\} 100$

where $C$ (i.e., control) $=$ total enzyme activity without inhibitor, and $\mathrm{T}$ (i.e., test sample) $=$ activity in the presence of test compound. ${ }^{\prime} C_{50}$ values (concentration that causes $50 \%$ inhibition of enzyme catalyzed reaction) of compounds were calculated using EZ-Fit Enzyme Kinetics Software (Perrella Scientific Inc. Amherst, USA).

\section{Butyrylcholinesterase (BChE) inhibitory assay}

BChE inhibition activity was estimated according to the method of Ellman et al [15]. The reaction mixture $(10 \mu \mathrm{L})$ contained $60 \mu \mathrm{L}$ of $\mathrm{Na}_{2} \mathrm{HPO}_{4}$ buffer $(50 \mathrm{mM}, \mathrm{pH} 7.7) ; 10 \mu \mathrm{L}$ of test compound $\left(0.5 \mathrm{mM}^{-1}\right.$ well $\left.^{-1}\right)$ and $10 \mu \mathrm{L}$ of BchE (0.5 unit well 1). After mixing, it was pre- read at $405 \mathrm{~nm}$ and then pre-incubated for 10 minutes at $37^{\circ} \mathrm{C}$. The reaction was initiated by addition of $10 \mu \mathrm{L}(0.5$ $\mathrm{mM}$ well $^{-1}$ ) of butyrylthiocholine iodide (substrate), followed by $10 \mu \mathrm{L}$ of DTNB $(0.5 \mathrm{mM}$ well $\left.^{-1}\right)$. After $15 \mathrm{~min}$ of incubation at $37^{\circ} \mathrm{C}$, absorbance was measured at $405 \mathrm{~nm}$. All experiments were carried out with their respective controls in triplicate. Eserine $(0.5 \mathrm{mM}$ well $^{-1}$ ) was used as positive control. Inhibition (\%) was calculated as in Eq 3.

Inhibition $(\%)=\{(C-T) / C\} 100$

where $C$ (i.e., control) = total enzyme activity without inhibitor, and $\mathrm{T}$ (i.e., test sample) = activity in the presence of test compound. ${ } \mathrm{C}_{50}$ values (concentration at which there is $50 \%$ enzyme inhibition) of compounds were calculated 
using EZ-Fit Enzyme kinetics software (Perella Scientific Inc. Amherst, USA). Results are expressed as mean of triplicate \pm SEM). Ciprofloxacin was used as standard.

\section{Antibacterial assay}

This was performed in sterile 96-wells micro plates under aseptic environments. The method is based on the principle that microbial cell number increases as the microbial growth proceeds in a log phase, which results in increased absorbance of the broth medium [16, 17]. Three gram-negative (Escherichia coli, Pseudomonas aeruginosa and Salmonella typhi) and two gram-positive bacteria (Bacillus subtilis, Staphylococcus aureus) maintained on agar medium were tested against the compounds. Test samples in suitable solvents and dilutions were pipetted into wells $(20 \mu \mathrm{g} /$ well) along with $180 \mu \mathrm{L}$ of freshly maintained overnight bacterial culture, after suitable dilution with fresh nutrient broth and incubated at $37{ }^{\circ} \mathrm{C}$ for $16-24 \mathrm{~h}$. Absorbance was measured at $540 \mathrm{~nm}$ using microplate reader, before and after incubation, and the difference was noted as an index of bacterial growth. The percent inhibition was calculated as in Eq 4.

Inhibition $(\%)=\{100(X-Y)\} / X$

where $X$ is the absorbance in control with bacterial culture and $Y$ is the absorbance of test sample.

\section{Hemolytic activity assay}

Hemolytic activities of the compounds were studied by the reported method $[18,19]$. Freshly obtained heparinized human blood $(3 \mathrm{~mL})$ was collected from volunteers after consent and counseling. The blood was centrifuged for $5 \mathrm{~min}$ at $1000 \mathrm{~g}$ and the plasma was discarded. The cells were then washed three times with $5 \mathrm{~mL}$ of chilled $\left(4^{\circ} \mathrm{C}\right)$ sterile isotonic phosphate-buffered saline (PBS, $\mathrm{pH}$ 7.4). The concentration of erythrocytes was maintained at $10^{8}$ cells per $\mathrm{mL}$ for each assay. Hundred $\mu \mathrm{L}$ of each compound was separately mixed with erythrocytes $\left(10^{8}\right.$ cells $/ \mathrm{mL}$ ), and incubated for $35 \mathrm{~min}$ at $37{ }^{\circ} \mathrm{C}$, followed by agitation after $10 \mathrm{~min}$. Thereafter the samples were placed on ice for $5 \mathrm{~min}$ and then centrifuged for $5 \mathrm{~min}$ at $1000 \mathrm{x} \mathrm{g}$. Supernatant samples $(100 \mu \mathrm{L})$ were taken from each tube and diluted 10 times with chilled $\left(4^{\circ} \mathrm{C}\right)$ PBS. Triton X-100 $(0.1 \% \mathrm{v} / \mathrm{v})$ was used as positive control, while phosphate buffered saline (PBS) was used as negative control. Absorbance was read at $576 \mathrm{~nm}$ using $\mu$ Quant (Bioteck,
USA). The \% of lysed RBCs for each sample was calculated.

\section{Docking methodology}

To predict the bioactive conformations, various compounds (ligands) were docked into the binding pockets of the selected proteins (enzymes) by using the default parameters of MOE-Dock program. Prior to docking, the protein molecules of a-glucosidase, LOX and BChE were retrieved from Protein Data Bank having PDB ID codes of $3 \mathrm{NO} 4$ (Resolution: 2.02A), 1IK3

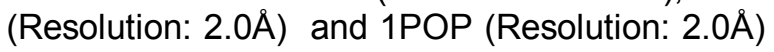
respectively. All the water molecules were removed from receptor proteins and 3D protonation was carried out by using the Protonate 3D Option. The energies of protein molecules were minimized by using the default parameters of MOE 2009-10 energy minimization algorithm (gradient: 0.05, Force Field: MMFF94X). Then all the ligands were docked into the binding pockets (selective residues/amino acids as shown in the docking images) of the above mentioned proteins using Triangular Matching docking method. Re-docking procedure was also applied to validate the docking protocol. Each complex was analyzed for the type of interactions; bond distances and their 3D images were taken.

\section{Statistical analysis}

All the measurements were carried out in triplicate and presented as mean \pm SEM. Statistical analysis was performed using Microsoft Excel 2010.

\section{RESULTS}

\section{Enzyme inhibitory activity}

In vitro screening of the synthesized compounds against $\alpha$-glucosidase, BChE and LOX revealed that these molecules exhibited variable inhibitory potential as shown by their $\mathrm{IC}_{50}$ values (Table-1). The compound 3-\{[5-(pentylsulfanyl)-1,3,4oxadiazol-2-yl]methyl\}-1H-indole $(\mathbf{6 g})$ showed inhibitory potential against a-glucosidase with $\mathrm{IC}_{50}$ value of $17.11 \pm 0.02 \mu \mathrm{g} / \mathrm{mL}$ as against acarbose with $\mathrm{IC}_{50}$ of $38.25 \pm 0.12 \mu \mathrm{g} / \mathrm{mL}$. Compounds 3-\{[5-(heptylsulfanyl)-1,3,4oxadiazol-2-yl]methyl\}-1H-indole (6h), 3-(\{5-[(4fluorobenzyl)sulfanyl]-1,3,4-oxadiazol-2-

yl\}methyl)-1H-indole (6s) and 3-(\{5-[(2methylbenzyl)sulfanyl]-1,3,4-oxadiazol-2-

yl\}methyl)-1H-indole (6t) produced inhibition activities against $\alpha$-glucosidase with $\mathrm{IC}_{50}$ values of $49.33 \pm 0.07,87.55 \pm 0.74$ and $98.81 \pm 0.69$ 
$\mu \mathrm{g} / \mathrm{mL}$ respectively. Compounds 3-\{[5-(isopropylsulfanyl)-1,3,4-oxadiazol-2-yl]methyl\}-1 Hindole (6d), 3-\{[5-(allylsulfanyl)-1,3,4-oxadiazol-2yl]methyl $\}-1 \mathrm{H}$-indole $(6 \mathrm{i})$ and $3-(\{5-[(3-$ phenylethyl)sulfanyl]-1,3,4-oxadiazol-2-

yl\}methyl)-1H-indole (6I) showed no inhibitory activity against the enzyme. The compound 3(\{5-[(4-chlorobenzyl)sulfanyl]-1,3,4-oxadiazol-2-

yl\}methyl)-1H-indole (6p) showed inhibitory potential against $\mathrm{BChE}$ with $\mathrm{IC}_{50}$ value of $31.0 \pm$ $0.73 \mu \mathrm{g} / \mathrm{mL}$ relative to the standard Eserine $\left(\mathrm{IC}_{50}\right.$ $=0.85 \pm 0.01 \mu \mathrm{g} / \mathrm{mL})$. Compounds 3- $(\{5-[(2-$ chlorobenzyl)sulfanyl]-1,3,4-oxadiazol-2-

yl\}methyl)-1H-indole (6n) and $3-(\{5-[(4-$ chlorobenzyl)sulfanyl]-1,3,4-oxadiazol-2-

yl\}methyl)-1H-indole $(\mathbf{6 p})$ had $\mathrm{IC}_{50}$ values $31.4 \pm$ 1.03 and $48.7 \pm 2.98 \mu \mathrm{g} / \mathrm{mL}$ against LOX, relative to standard baicalein $\left(\mathrm{IC}_{50}=22.4 \pm 1.30 \mu \mathrm{g} / \mathrm{mL}\right)$.

\section{Antibacterial activity}

The synthesized compounds exhibited variable antibacterial activities. For example, compounds 3-\{[5-(propylsulfanyl)-1,3,4-oxadiazol-2-

yl]methyl\}-1H-indole $\quad(6 \mathrm{c}), \quad 3$ - $\{[5$-(isopropylsulfanyl)-1,3,4-oxadiazol-2-yl]methyl\}-1Hindole (6d) and 3-(\{5-[(4-fluorobenzyl)sulfanyl]1,3,4-oxadiazol-2-yl\}methyl)-1H-indole produced antibacterial potential against $S$. typhi, with MIC values of $10.26 \pm 1.08,10.06 \pm 1.66$ and $10.64 \pm 2.08 \mu \mathrm{M}$ respectively. Some compounds such as 3-\{[5-(iso-propylsulfanyl)1,3,4-oxadiazol-2-yl]methyl\}-1H-indole (6d), 3(\{5-[(4-bromobenzyl)sulfanyl]-1,3,4-oxadiazol-2yl\}methyl)-1H-indole $\quad(6 \mathbf{r})$ and 3-(\{5-[(4fluorobenzyl)sulfanyl]-1,3,4-oxadiazol-2-

yl\}methyl)-1H-indole, showed inhibition against E. coli, (6s) with MIC values $9.75 \pm 1.33,9.26 \pm$ 2.76 and $9.12 \pm 2.15 \mu \mathrm{M}$ respectively, as against standard ciprofloxacin (MIC $=7.89 \pm 1.54 \mu \mathrm{M})$. The compound 3-\{[5-(allylsulfanyl)-1,3,4oxadiazol-2-yl]methyl\}-1H-indole (6i) had MIC value of $10.43 \pm 2.29 \mu \mathrm{M}$ against $P$. aeruginosa. Compounds 3-\{[5-(butylsulfanyl)-1,3,4-oxadiazol2-yl]methyl\}-1H-indole (6f) and 3-(\{5-[(4bromobenzyl)sulfanyl]-1,3,4-oxadiazol-2-

yl\}methyl)-1H-indole (6r) showed inhibitory potential against $B$. subtilis with MIC values of $9.75 \pm 3.65$ and $9.96 \pm 2.95 \mu \mathrm{M}$ respectively. Furthermore, compounds 3-\{[5-(propylsulfanyl)1,3,4-oxadiazol-2-yl]methyl\}-1H-indole (6c), 3$\{[5$-(iso-propylsulfanyl)-1,3,4-oxadiazol-2-

yl]methyl\}-1H-indole (6d) showed inhibition potential against $S$. aureus with MIC values 9.14 \pm 2.67 and $9.00 \pm 4.12 \mu \mathrm{M}$, which are comparable to that of ciprofloxacin $(8.11 \pm 2.12$ $\mu \mathrm{M})$ (Table 2).

Table 1: Enzyme Inhibition activities of the S-alkylated/aralkylated 2-(1H-indol-3-ylmethyl)-1,3,4-oxadiazole-5thiol derivatives (6a-u)

\begin{tabular}{|c|c|c|c|}
\hline \multirow{2}{*}{ Code } & $\alpha$-Glucosidase & Butyrylcholinestrase (BChE) & $\begin{array}{l}\text { Lipoxygenase } \\
\text { (LOX) }\end{array}$ \\
\hline & $\begin{array}{c}I C_{50} \\
(\mu \mathrm{g} / \mathrm{mL})\end{array}$ & $\begin{array}{c}I C_{50} \\
(\mu \mathrm{g} / \mathrm{mL})\end{array}$ & $\begin{array}{c}I C_{50} \\
(\mu \mathrm{g} / \mathrm{mL})\end{array}$ \\
\hline $6 a$ & $294.1 \pm 1.15$ & $262 \pm 1.38$ & - \\
\hline $6 b$ & $165.2 \pm 1.84$ & $140 \pm 1.56$ & - \\
\hline $6 c$ & $135.6 \pm 0.34$ & $65.0 \pm 0.45$ & - \\
\hline $6 d$ & - & $245 \pm 0.61$ & - \\
\hline $6 e$ & $228.5 \pm 1.81$ & $134 \pm 0.29$ & $>500$ \\
\hline $6 f$ & $117.3 \pm 1.01$ & $146 \pm 0.57$ & $>500$ \\
\hline $6 \mathrm{~g}$ & $17.11 \pm 0.02$ & $345 \pm 1.78$ & - \\
\hline $6 h$ & $49.33 \pm 0.07$ & $343 \pm 1.89$ & $>500$ \\
\hline $6 i$ & - & $434 \pm 0.34$ & - \\
\hline $6 \mathbf{j}$ & $>400$ & $66.1 \pm 2.18$ & $136 \pm 0.99$ \\
\hline $6 k$ & $463.9 \pm 2.48$ & $340 \pm 3.90$ & $>500$ \\
\hline 61 & - & $142 \pm 2.65$ & $>500$ \\
\hline $6 m$ & $98.25 \pm 0.75$ & $324 \pm 1.45$ & $218 \pm 1.23$ \\
\hline $6 n$ & $474.2 \pm 1.83$ & $231 \pm 2.90$ & $31.4 \pm 1.03$ \\
\hline 60 & $443.9 \pm 1.25$ & $52.0 \pm 2.45$ & $>500$ \\
\hline $6 p$ & $431.7 \pm 1.87$ & $31.0 \pm 0.73$ & $48.7 \pm 2.98$ \\
\hline $6 q$ & $319.1 \pm 1.96$ & $160 \pm 0.36$ & $>500$ \\
\hline $6 r$ & $266.8 \pm 1.95$ & $134 \pm 0.98$ & $>500$ \\
\hline $6 s$ & $87.55 \pm 0.74$ & $54.5 \pm 1.90$ & $135 \pm 1.09$ \\
\hline $6 t$ & $98.81 \pm 0.69$ & $70.0 \pm 3.16$ & $130 \pm 0.34$ \\
\hline $6 u$ & $>400$ & $154 \pm 0.43$ & $178 \pm 0.76$ \\
\hline Control & $\begin{array}{c}38.25 \pm 0.12 \\
\text { Acarbose }\end{array}$ & $\begin{array}{c}0.85 \pm 0.01 \\
\text { Eserine }\end{array}$ & $\begin{array}{l}22.4 \pm 1.30 \\
\text { Baicalein }\end{array}$ \\
\hline
\end{tabular}

Values are mean \pm SEM. $(n=3)$ 
Table 2: Antibacterial activities (\% inhibition) of S-alkylated/aralkylated 2-(1H-indol-3-ylmethyl)-1,3,4-oxadiazole5-thiols (6a-u)

\begin{tabular}{|c|c|c|c|c|c|}
\hline Inhibition (\%) & S. typhi (-) & E. coli (-) & P. aeruginosa (-) & B. subtilis (+) & S. aureus(+) \\
\hline $6 a$ & $56.73 \pm 0.18$ & $44.45 \pm 3.00$ & $40.83 \pm 0.71$ & $42.45 \pm 4.55$ & $56.35 \pm 0.75$ \\
\hline $6 b$ & $77.09 \pm 0.00$ & $70.50 \pm 2.23$ & $75.75 \pm 0.67$ & $62.32 \pm 5.00$ & $68.30 \pm 0.10$ \\
\hline $6 c$ & $82.27 \pm 0.27$ & $82.00 \pm 0.36$ & $83.04 \pm 1.79$ & $64.00 \pm 0.55$ & $77.65 \pm 0.85$ \\
\hline $6 d$ & $79.95 \pm 2.50$ & $73.09 \pm 3.45$ & $75.17 \pm 2.08$ & $63.55 \pm 5.00$ & $67.25 \pm 1.35$ \\
\hline $6 e$ & $69.77 \pm 1.23$ & $49.82 \pm 4.55$ & $62.67 \pm 3.08$ & $55.73 \pm 4.27$ & $63.05 \pm 4.55$ \\
\hline $6 f$ & $64.09 \pm 2.81$ & $52.41 \pm 5.00$ & $70.88 \pm 3.79$ & $64.64 \pm 1.55$ & $58.85 \pm 2.45$ \\
\hline $6 g$ & $62.91 \pm 1.64$ & $53.05 \pm 2.77$ & $67.83 \pm 1.50$ & $55.09 \pm 3.00$ & $57.75 \pm 1.75$ \\
\hline $6 h$ & $59.09 \pm 1.00$ & $48.45 \pm 2.82$ & $49.46 \pm 1.38$ & $46.86 \pm 4.50$ & $63.75 \pm 0.05$ \\
\hline $6 i$ & $78.45 \pm 2.45$ & $72.05 \pm 0.41$ & $68.21 \pm 0.54$ & $68.32 \pm 0.41$ & $64.55 \pm 0.65$ \\
\hline $6 j$ & $71.64 \pm 0.73$ & $69.36 \pm 0.36$ & $67.33 \pm 3.50$ & $62.50 \pm 3.95$ & $67.65 \pm 0.05$ \\
\hline $6 \mathrm{k}$ & $70.77 \pm 1.59$ & $59.27 \pm 0.45$ & $67.17 \pm 1.33$ & $58.41 \pm 1.05$ & $62.00 \pm 3.60$ \\
\hline 61 & $65.32 \pm 1.41$ & $54.68 \pm 1.50$ & $57.04 \pm 2.21$ & $60.00 \pm 1.64$ & $56.90 \pm 0.50$ \\
\hline $6 m$ & $60.95 \pm 0.05$ & $52.00 \pm 0.27$ & $56.71 \pm 0.13$ & $55.86 \pm 1.23$ & $55.40 \pm 2.30$ \\
\hline $6 n$ & $65.23 \pm 1.23$ & $53.18 \pm 3.00$ & $52.00 \pm 3.75$ & $46.73 \pm 5.00$ & $51.80 \pm 1.10$ \\
\hline 60 & $56.68 \pm 0.50$ & $52.09 \pm 0.09$ & $54.67 \pm 0.42$ & $49.91 \pm 1.00$ & $52.65 \pm 1.55$ \\
\hline $6 p$ & $59.50 \pm 1.14$ & $51.00 \pm 1.91$ & $55.92 \pm 0.58$ & $54.09 \pm 2.64$ & $63.85 \pm 1.55$ \\
\hline $6 q$ & $59.64 \pm 1.18$ & $47.09 \pm 4.73$ & $57.38 \pm 1.54$ & $51.27 \pm 3.27$ & $66.35 \pm 1.65$ \\
\hline $6 r$ & $73.95 \pm 0.68$ & $65.41 \pm 0.23$ & $72.92 \pm 0.33$ & $67.23 \pm 1.41$ & $64.45 \pm 0.45$ \\
\hline $6 s$ & $65.41 \pm 0.23$ & $68.36 \pm 0.18$ & $74.38 \pm 1.21$ & $49.95 \pm 2.77$ & $57.05 \pm 0.45$ \\
\hline $6 t$ & $72.00 \pm 0.45$ & $62.27 \pm 1.55$ & $65.75 \pm 0.58$ & $58.68 \pm 0.50$ & $64.50 \pm 1.20$ \\
\hline $6 u$ & $64.00 \pm 0.55$ & $48.18 \pm 1.36$ & $53.25 \pm 0.25$ & $56.55 \pm 3.55$ & $50.35 \pm 2.65$ \\
\hline $\begin{array}{l}\text { Ciprofloxacin } \\
\text { MIC }(\boldsymbol{\mu})\end{array}$ & $91.90 \pm 3.00$ & $92.56 \pm 0.05$ & $91.00 \pm 2.13$ & $91.45 \pm 0.45$ & $90.80 \pm 1.90$ \\
\hline $6 a$ & $12.54 \pm 0.96$ & - & - & - & $14.65 \pm 0.74$ \\
\hline $6 b$ & $14.35 \pm 1.21$ & $10.23 \pm 1.65$ & $12.70 \pm 1.97$ & $13.33 \pm 1.56$ & $10.98 \pm 1.50$ \\
\hline $6 c$ & $10.26 \pm 1.08$ & $11.21 \pm 2.12$ & $14.42 \pm 2.17$ & $13.24 \pm 1.88$ & $9.14 \pm 2.67$ \\
\hline $6 d$ & $10.06 \pm 1.66$ & $9.75 \pm 1.33$ & $11.22 \pm 1.64$ & $10.89 \pm 2.34$ & $9.00 \pm 4.12$ \\
\hline $6 e$ & $11.43 \pm 1.97$ & - & $14.67 \pm 2.41$ & $14.37 \pm 3.00$ & $12.44 \pm 2.64$ \\
\hline $6 f$ & $13.62 \pm 0.59$ & $16.53 \pm 0.54$ & $12.78 \pm 1.48$ & $9.75 \pm 3.65$ & $15.75 \pm 2.29$ \\
\hline $6 g$ & $12.62 \pm 1.11$ & $15.46 \pm 0.76$ & $12.83 \pm 2.09$ & $15.41 \pm 2.00$ & $12.89 \pm 1.41$ \\
\hline $6 \mathrm{~h}$ & $15.68 \pm 0.45$ & - & - & - & $12.59 \pm 0.33$ \\
\hline $6 i$ & $11.83 \pm 2.00$ & $10.63 \pm 1.39$ & $10.43 \pm 2.29$ & $10.75 \pm 2.61$ & $11.44 \pm 2.14$ \\
\hline $6 j$ & $13.16 \pm 0.66$ & $13.57 \pm 0.66$ & $13.52 \pm 1.43$ & $12.18 \pm 1.78$ & $10.10 \pm 2.00$ \\
\hline $6 \mathrm{k}$ & $14.02 \pm 0.98$ & $12.74 \pm 1.58$ & $13.76 \pm 2.71$ & $11.51 \pm 1.84$ & $13.85 \pm 1.67$ \\
\hline 61 & $11.90 \pm 1.60$ & $13.53 \pm 1.93$ & $16.08 \pm 3.72$ & $13.40 \pm 1.47$ & $10.59 \pm 0.74$ \\
\hline $6 m$ & $15.18 \pm 3.23$ & $14.99 \pm 0.67$ & $18.02 \pm 5.00$ & $12.70 \pm 2.53$ & $10.32 \pm 1.55$ \\
\hline $6 n$ & $15.94 \pm 0.62$ & $18.25 \pm 0.22$ & $18.81 \pm 5.00$ & $14.00 \pm 1.22$ & $18.81 \pm 5.00$ \\
\hline 60 & $16.82 \pm 1.00$ & $17.02 \pm 1.81$ & $16.64 \pm 4.42$ & $13.89 \pm 2.03$ & $16.64 \pm 4.42$ \\
\hline $6 p$ & $12.01 \pm 2.45$ & $16.66 \pm 1.00$ & $16.30 \pm 3.75$ & $12.24 \pm 2.15$ & $16.30 \pm 3.75$ \\
\hline $6 q$ & $12.66 \pm 1.03$ & - & $13.33 \pm 1.75$ & $15.50 \pm 3.00$ & $13.33 \pm 1.75$ \\
\hline $6 r$ & $12.08 \pm 1.38$ & $9.26 \pm 2.76$ & $15.64 \pm 2.33$ & $9.96 \pm 2.95$ & $15.64 \pm 2.33$ \\
\hline $6 s$ & $10.64 \pm 2.08$ & $9.12 \pm 2.15$ & $11.34 \pm 2.25$ & $12.71 \pm 2.00$ & $11.34 \pm 2.25$ \\
\hline $6 t$ & $13.95 \pm 0.22$ & $12.37 \pm 1.32$ & $14.09 \pm 2.50$ & $12.56 \pm 1.24$ & $14.09 \pm 2.50$ \\
\hline $6 u$ & $11.13 \pm 2.12$ & $14.99 \pm 0.67$ & $17.30 \pm 3.08$ & $18.51 \pm 4.00$ & $17.30 \pm 3.08$ \\
\hline Ciprofloxacin & $8.04 \pm 0.15$ & $7.89 \pm 1.54$ & $8.54 \pm 1.76$ & $7.29 \pm 2.67$ & $8.11 \pm 2.12$ \\
\hline
\end{tabular}

Minimum inhibitory concentration (MIC) was measured with suitable dilutions (5-30 $\mu \mathrm{g} / \mathrm{well}$ ) and results were calculated using EZ-Fit Perrella Scientific Inc. Amherst USA software. Values are mean \pm SEM $(n=3)$

\section{Hemolytic activity}

The lowest hemolytic activity was exhibited by the compound $6 \mathrm{c}(1.50 \%)$ which was similar to the negative control (PBS). Compounds $6 f(5.37$ $\%), 6 \mathrm{~m}(6.88 \%)$, $6 \mathbf{e}(6.94 \%)$ and $6 \mathbf{l}(7.63 \%)$ also exhibited low hemolytic activities. The highest hemolytic activity was produced by compound $6 u(20.56 \%)$ but this was lower than the activity of the positive control (Triton-X-100) (Table 3).

\section{Molecular docking}

Molecular docking studies confirmed the antienzymatic activities of all the compounds Figure1. $2 D$ and $3 D$ docked pictures of some more potent compounds are given here. 
Table 3: Hemolytic activities of S-alkylated/aralkylated 2-(1H-indol-3yl-methyl)-1,3,4-oxadiazole-5-thiols (6a-u)

\begin{tabular}{|c|c|c|c|c|c|}
\hline Code & $\%$ Hemolysis & Compound & $\%$ Hemolysis & Compound & $\%$ Hemolysis \\
\hline $6 a$ & 11.13 & 6h & 7.44 & 60 & 7.19 \\
\hline $6 b$ & 18.94 & $6 i$ & 13.44 & $6 p$ & 9.75 \\
\hline $6 c$ & 1.50 & $6 j$ & 17.56 & $6 q$ & 10.81 \\
\hline $6 d$ & 14.00 & $6 \mathrm{k}$ & 10.31 & $6 r$ & 10.94 \\
\hline $6 e$ & 6.94 & 61 & 7.63 & $6 s$ & 9.38 \\
\hline $6 f$ & 5.37 & $6 m$ & 6.88 & $6 t$ & 11.50 \\
\hline $6 g$ & 10.25 & $6 n$ & 8.25 & $6 u$ & 20.56 \\
\hline PBS & 00.00 & Triton-X & 100.00 & & \\
\hline
\end{tabular}

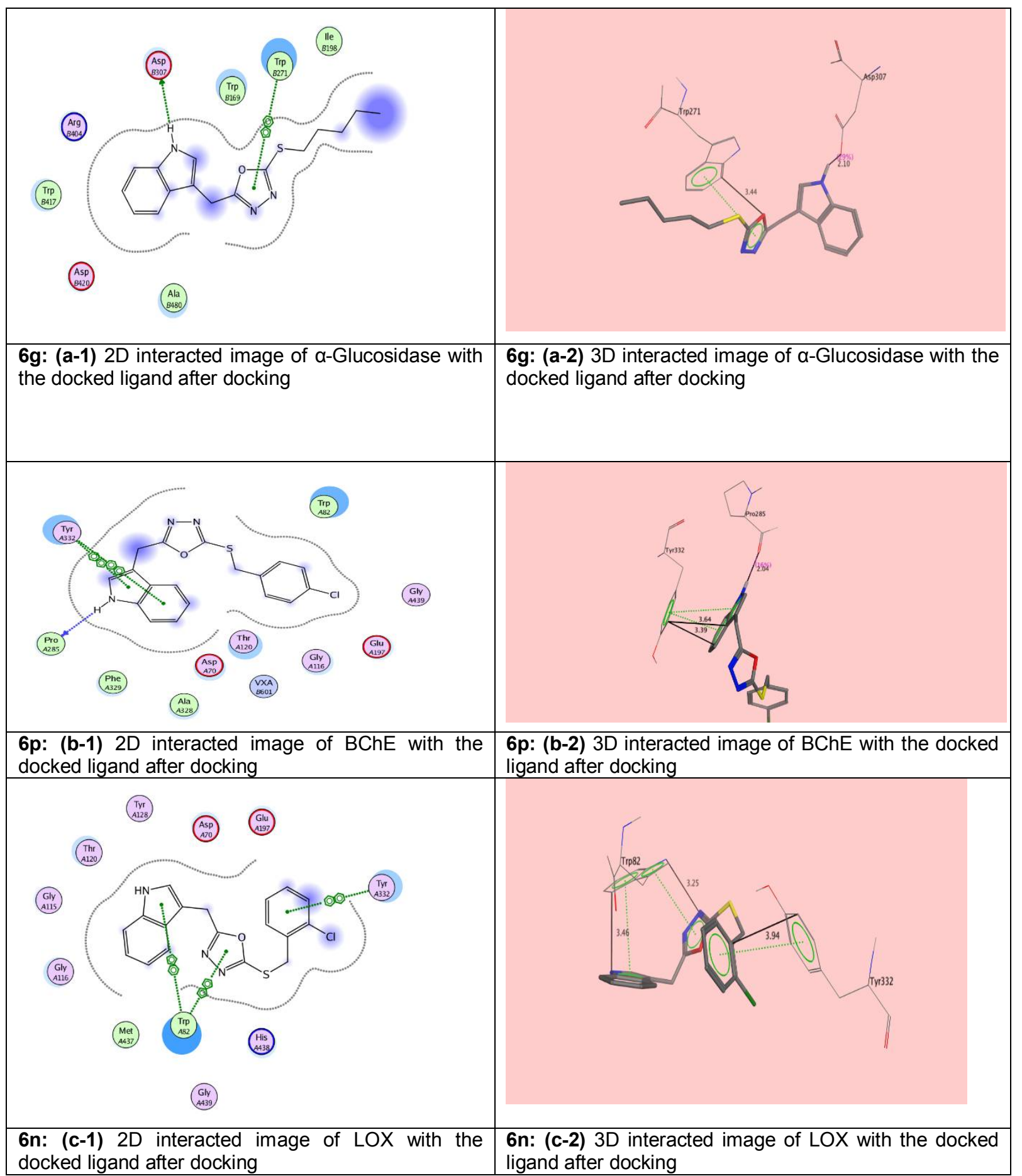

Figure 1: Binding modes of some of the compounds against $\alpha$ - glucosidase, BChE and LOX 


\section{DISCUSSION}

Compounds bearing oxadiazole moieties have been previously synthesized by our research group and their biological activities were shown to be related to structural modifications in the molecules [20, 21]. The synthesis of these $5-(1 \mathrm{H}-$ indol-3yl-methyl)-1,3,4-oxadiazole-2-thiols (6a-u) in the present study is a part of our continued efforts to generate new molecules with biological activities. Our results revealed that the $5-(1 \mathrm{H}-$ indol-3yl-methyl)-1,3,4-oxadiazole-2-thiol

derivatives (6a-6u) had variable inhibitory potencies against a-glucosidase, BChE and LOX. As expected, the compounds with comparatively more inhibitory activities were those that fitted more closely into the active sites of the enzymes inhibited. Compound $3-\{[5-$ (pentylsulfanyl)-1,3,4-oxadiazol-2-yl]methyl\}-1Hindole $(\mathbf{6 g})$ showed excellent inhibitory potential against $\alpha$-glucosidase, which was even better than the reference standard, acarbose. This molecule contains n-pentyl group substituted at the thiol position of the oxadiazole ring along with the indole moiety. These structural features probably conferred on the compound the best fit into the active site of the enzyme, which might be responsible for its high inhibitory potency against a-glucosidase. Similarly compounds 3-\{[5(heptylsulfanyl)-1,3,4-oxadiazol-2-yl]methyl\}-1Hindole (6h), 3-(\{5-[(4-fluorobenzyl) sulfanyl]-1,3,4oxadiazol-2-yl\}methyl)-1H-indole (6s) and 3-(\{5[(2-methylbenzyl)sulfanyl] -1,3,4-oxadiazol-2yl\}methyl)-1H-indole (6t) also yielded relatively high inhibition potential against the enzyme. These molecules contain n- heptyl, 4-florobenzyl and 2-methylbenzyl substituents respectively at the thiol positions, which might be responsible for their being able to fit into the active site of $\alpha$ glucosidase.

All the synthesized derivatives exhibited moderate to weak inhibition against BChE, whereas, comparatively better inhibition potential was shown by the compound $3-(\{5[(4-$ chlorobenzyl)sulfanyl]-1,3,4-oxadiazol-2-

yl\}methyl)-1H-indole (6p) against this enzyme, when compared to the standard Eserine. Compound $6 \mathrm{p}$ has S-substituted 4-chlorobenzyl group which might have been responsible for its better fitting into the active site of BChE [22]. Compounds 3-(\{5-[(2-chlorobenzyl)sulfanyl]1,3,4-oxadiazol-2-yl\}methyl)-1H-indole (6n) and 3-(\{5-[(4-chlorobenzyl)sulfanyl]-1,3,4-oxadiazol2-yl\}methyl)-1H-indole (6p) which possess 2chlorobenzyl and 4-chlorobenzyl substituents respectively, showed good inhibitory potential against LOX, relative to standard Baicalein [23].
All the synthesized compounds exhibited variable antibacterial potential. It has been reported earlier that slight modifications in structure may affect antibacterial potential [24]. In this study we made changes at the thiol position of the parent indole-containing oxadiazole molecule (4) by substituting different alkyl/aralkyl groups to evaluate their antibacterial profile [25]. For example, the compounds $3-\{[5-($ propylsulfanyl)1,3,4-oxadiazol-2-yl]methyl\}-1H-indole (6c), 3$\{[5-($ iso-propylsulfanyl)-1,3,4-oxadiazol-2-

yl]methyl $\}-1 \mathrm{H}$-indole $(6 \mathrm{~d}) \quad$ and $3-(\{5-[(4-$ fluorobenzyl)sulfanyl]-1,3,4-oxadiazol-2-

yl\}methyl)-1H-indole (6s) which contain n-propyl, iso-propyl and 4-flourobenzyl groups respectively, at the thiol positions, showed good antibacterial activities against $S$. typhi.

The compound 3-\{[5-(iso-propylsulfanyl)-1,3,4oxadiazol-2-yl]methyl\}-1H-indole (6d) and 3-(\{5[(4-bromobenzyl)sulfanyl]-1,3,4-oxadiazol-2yl\}methyl)-1H-indole (6r) also showed good activities against $E$. coli along with $3-(\{5-[(4-$ fluorobenzyl)sulfanyl]-1,3,4-oxadiazol-2-

yl\}methyl)-1H-indole (6s), and their MIC values were close to that of standard ciprofloxacin. However the antibacterial activities of the compounds against $P$. aeruginosa were comparatively lower. Indeed only the compound 3-\{[5-(allylsulfanyl)-1,3,4-oxadiazol-2-yl]methyl\}$1 \mathrm{H}$-indole (6i) exhibited appreciable activity against this strain. The allylic group attached at the thiol position of the parent molecule (4) might be responsible for this activity. Compounds 3-\{[5(butylsulfanyl)-1,3,4-oxadiazol-2-yl]methyl\}-1Hindole (6f) and 3-(\{5-[(4-bromobenzyl)sulfanyl]1,3,4-oxadiazol-2-yl\}methyl)-1H-indole showed very good antibacterial potential against $B$. subtilis, which were very close to that of the standard drug. This might be attributed to the Ssubstituted n-butyl and 4-bromobenzyl groups in these compounds. Furthermore, compounds 3$\{[5-($ propylsulfanyl)-1,3,4-oxadiazol-2-yl]methyl\}1H-indole (6c), 3-\{[5-(iso-propylsulfanyl)-1,3,4oxadiazol-2-yl]methyl\}-1H-indole (6d) also yielded very good inhibition potential against $S$. aureus comparable to ciprofloxacin.

The compound 3-\{[5-(pentylsulfanyl)-1,3,4oxadiazol-2-yl]methyl\}-1H-indole $(\mathbf{6 g})$ which exhibited excellent inhibitory potential against $\alpha$ glucosidase (even better than reference standard) also exhibited comparatively lower \% hemolytic activity. This is a desirable characteristic. In addition, compound 3-\{[5(propylsulfanyl)-1,3,4-oxadiazol-2-yl]methyl\}-1Hindole (6c) which produced excellent antibacterial effect on $S$. aureus, had the lowest \% hemolytic activity. 
Other compounds that exhibited comparable antibacterial activity against $S$. aureus, such as 3-\{[5-(iso-propylsulfanyl)-1,3,4-oxadiazol-2yl]methyl\}-1H-indole (6d) also showed low \% hemolytic activity. The compounds 3-\{[5(butylsulfanyl)-1,3,4-oxadiazol-2-yl]methyl\}-1Hindole (6f), 3-(\{5-[(4-bromobenzyl)sulfanyl]-1,3,4oxadiazol-2-yl\}methyl)-1H-indole (6r) showed least values of \% hemolytic activity and good values of antibacterial activity against $B$. subtilis. The compound 3-(\{5-[(4-fluorobenzyl)sulfanyl]1,3,4-oxadiazol-2-yl\}methyl)-1H-indole (6s), also exhibited comparatively lower values of \% hemolytic activity and good antibacterial activity against both $S$. typhi and E. coli.

On the basis of these results, it seems reasonable to assume that these synthesized thiol derivative compounds are either non-toxic or at most, only moderately so. Thus they may be suitable drug candidates for further investigations.

In order to test the validity of our enzyme inhibition results, the co-crystallized ligands of the enzymes were extracted and then re-docked into the binding pockets of the receptors. In all cases, RMSD values between docked and cocrystallized ligands were less than $2 \mathrm{~A}^{\circ}$ which indicated the reliability of docking method. Thus our protocol can be used for further studies. Almost all the synthesized derivatives were computationally docked against a-glucosidase, $\mathrm{BChE}$ and lipoxygenase to explore the binding modes of all the ligands.

The results were very much in favor of the experimental data. For example, 3-\{[5(pentylsulfanyl)-1,3,4-oxadiazol-2-yl]methyl\}-1Hindole $(\mathbf{6 g})$ showed interactions with two active site amino acid residues of the target protein. Amino acid Asp B307 showed a hydrogen bond acceptor (polar) interaction with $-\mathrm{NH}$ group of indole moiety with a bond distance of 2.10 angstroms, while Trp307 showed arene-arene interaction with oxadiazole ring having a bond distance of 3.44 angstroms. The other residues present in the nearby vicinity of the ligand were Trp B269, Arg B404 and IleB198.

Two amino acid residues interacted with $3-(\{5-$ [(4-chlorobenzyl)sulfanyl]-1,3,4-oxadiazol-2yl\}methyl)-1H-indole (6p). Tyr A332 showed two arene- arene interactions with indole moiety showing bond length of 3.39 angstroms with six membered ring of indole moiety on one hand, and 3.34 angstroms with five membered ring of indole moiety on the other hand. Other residues found in close contact with the ligand were Thr A120, and Asp A70. Two amino acid residues interacted with compound 3-(\{5-[(2chlorobenzyl)sulfanyl]-1,3,4-oxadiazol-2-

yl\}methyl)-1H-indole (6n) as Trp A82 showing two arene-arene interactions with five membered ring of indole moiety having bond length of 3.46 angstroms and with other five membered ring of oxadiazole with bond length of 3.25 angstroms. Tyr A332 showed arene-arene interaction with 2-chlorobenzyl ring having bond length of 3.94 angstroms. Other residues that existed in the near most region of the ligand were Met A437, His A438, and Asp A70.

\section{CONCLUSION}

All the compounds exhibited variable antienzymatic potential which were also supported by the molecular docking results. Compound 3$\{[5-($ pentylsulfanyl)-1,3,4-oxadiazol-2-yl]methyl\}-

$1 \mathrm{H}$-indole $(\mathbf{6 g})$ showed excellent anti-enzymatic activity against $\alpha$-glucosidase, which was superior to that of the standard Baicalein. Their anti-bacterial activities revealed that the synthesized derivatives displayed moderate to very good to activities. Compounds $3-\{[5-$ (propylsulfanyl)-1,3,4-oxadiazol-2-yl]methyl\}-1Hindole (6c), 3-\{[5-(iso-propylsulfanyl)-1,3,4oxadiazol-2-yl]methyl\}-1H-indole $\quad(6 \mathrm{~d}), \quad 3-\{[5-$ (butylsulfanyl)-1,3,4-oxadiazol-2-yl] methyl\}-1Hindole (6f), 3-(\{5-[(4-bromobenzyl)sulfanyl]-1,3,4oxadiazol-2-yl\}methyl)-1H-indole (6r) and 3-(\{5[(4-fluorobenzyl)sulfanyl]-1,3,4-oxadiazol-2-

yl\}methyl)-1H-indole (6s) showed MIC values very close to that of standard ciprofloxacin. These compounds also showed least to moderate \% hemolytic activities. These results suggest that the 2-(1H-indol-3-ylmethyl)-1,3,4oxadiazole-5-thiol derivatives, due to their multiple functional groups, possess remarkable anti-enzymatic and antibacterial potential with acceptable cytotoxicity levels. Thus they may be useful agents for development of new antidiabetic drugs and anti-inflammatory agents.

\section{DECLARATIONS}

\section{Conflict of Interest}

No conflict of interest associated with this work.

\section{Contribution of Authors}

The authors declare that this work was done by the authors named in this article and all liabilities pertaining to claims relating to the content of this article will be borne by them. 


\section{REFERENCES}

1. Vijay VD, Nitin VB. Synthesis of 2-Substituted-1,3,4Oxadiazole Derivatives. Inter J Chem Env Pharm Res 2011; 2(1): 1-4.

2. Dhansay $D$, Alok $P$, Sivakumar $T$, Rajavel $R$, Dubey $R D$. Synthesis, Fungicidal Activity, and 3D-QSAR of Pyridazinone-Substituted 1,3,4-Oxadiazoles and 1,3,4Thiadiazoles. Inter J Chem Tech Res 2010; 2(3): 1397 1412.

3. Ryhanen RJ. Pseudocholinesterase activity in some human body fluids. J Pharmacol 1983; 14: 459-60.

4. Dave KR, Syal AR, Kayare SS. Tissue Cholinesterases. A comparative study of their kinetic properties. Z Naturforsch 2000; 55: 100-8.

5. Silver A. The biology of cholinesterases. New York: Elsevier Agricultural Research Council Institute; 2000.

6. Lebovitz HE. Alpha-glucosidase inhibitors, Endocrinology and Metabolism. Clin N Am 1997; 26: 539-551.

7. Roussaki $M$, Kontogiorgis $C A$, Litina $D H$, Hamilakis $S$, Detsi A. A novel synthesis of 3-aryl coumarins and evaluation of their antioxidant and lipoxygenase inhibitory activity. Bioorgan Med Chem Lett 2010; 20 : 3889-3892.

8. Shahzadi T, Abbasi MA, Aziz-ur-Rehman, Riaz T, Khan KM, Ashraf M, Afzal I, Akhtar MN, and Ajaib $M$. Antioxidant and Lipoxygenase Inhibiting New Iridoid Glucosides from Caryopteris odorata. Nat. Prod. Res. 2013; 27(4-5): 302-313.

9. Abbasi MA, Akhtar A, Aziz-ur-Rehman, Nafeesa K, Siddiqui SZ, Khan MK, Ashraf M, Ejaz SA. Synthesis, structural charcterization and enzyme inhibition studies on 5-(2-nitrostyryl)-1,3,4-oxadizole-2-thiol derivatives. J Chil Chem Soc 2013; 58: 2186-2190.

10. Abbasi MA, Shahzad B, Aziz-ur-Rehman, Nafeesa $K$, Rasool S, Ashraf M, Ejaz SA, Ismail H, Mirza B. Synthesis, Spectral Characterization and Bioactivity Studies of Some S-Substituted Derivatives of 5-(4Chlorophenyl)-1,3,4-Oxadiazole-2-Thiol.World J Pharm Sci 2014; 2(1): 32-40.

11. Tappel AL. The mechanism of the oxidation of unsaturated fatty acid catalyzed by hematin compounds. Arch Biochem Biophy 1953; 44(2): 378-395.

12. Evans AT. Actions of cannabis constituents on enzymes of arachidonate metabolism: anti-inflammatory potential. Biochem Pharmacol 1987; 36: 2035-2037.

13. Baylac S, Racine P. Inhibition of 5-lipoxygenase by essential oils and other natural fragrant extracts. Int $J$ Aromatherp. 2003; 13: 138-142.

14. Pierre C, Roland R, Tremblay DJY. p-Nitrophenol- $\alpha-D$ Glucopyranoside as substrate for measurement of maltase activity in Human Semen. Clin Chem 1978; 24: 208-211.

15. Ellman GL, Courtney KD, Andres V, Featherstone RM. A new and rapid calorimetric determination of acetylcholinesterase activity. Biochem Pharmacol 1961; 7: 88-95.
16. Kaspady M, Narayanaswamy VK, Raju M, Rao GK. Synthesis, antibacterial activity of 2,4-disubstituted oxazoles and thiazoles as bioesters. Lett Drug Design Discov 2009; 6: 21-28.

17. Yang CR, Zang Y, Jacob MR, Khan SI, Zhang YJ, Li XC. Antifungal activity of C-27 steroidal saponins. Antimicrob Agents Chemotherap 2006; 50(5): 1710-1714.

18. Shahid M, Bukhari SA, Gul Y, Munir H, Anjum F, Zuber $M$, Jamil T, Zia KM. Graft polymerization of guar gum with acryl amide irradiated by microwaves for colonic drug delivery. Int J Biolog Macromol 2013; 62:172-179.

19. Zuber M, Tabasum S, Jamil T, Shahid M, Hussain R, Feras K, Bhatti K. Biocompatibility and microscopic evaluation of polyurethane-poly(methyl methacrylate)titanium dioxide based composites for dental applications. J App Polym Sci 2014; 131: doi: 10.1002/app.39806.20. Siddiqui S Z, Abbasi M A, Azizur-Rehman, Irshad M, Shahzad B, Ashra Mf, Ahmad I, Lodhi M A, Mirza B, Ismail $H$, Akhtar M N. Synthesis pharmacological evaluation, molecular docking and cytotoxic studies on some $\mathrm{N}$-substituted 5-[(4chlorophenoxy)methyl]-1,3,4-oxadiazole-2yl-2-sulfanyl acetamides. Indo Am. J. Pharm. Resh 2014; 4(8): 3603-3616

20. Siddiqui S Z, Aziz-ur-Rehman, Abbasi M A, Abbas N, Khan $K M$, Ashraf $M$, Ejaz $S$ A. Synthesis, Characterization and Biological Screening of $\mathrm{N}$ Substituted Derivatives of 5-Benzyl-1,3,4-oxadiazole2yl-2"-sulfanyl Acetamide. Pak. J. Pharm. Sci 2013; 26(3): 455-463.

21. Abbasi M A, Najm S, Aziz-ur-Rehman, Rasool S, Khan $K$ $M$, Ashraf M, Nasar R, Alam U. Evaluation of Sulfonamide Derivatives of Dagenan Chloride as Lipoxygenase and $\alpha$-Glucosidase Inhibitors. Trop. J. Pharm. Res. 2015; 14 (1): 47-54.

22. Abbasi M A, Akhtar A, Aziz-Ur-Rehman, Nafeesa K, Siddiqui S Z, Khan K M, Ashraf M, Ejaz S A. Synthesis, structural characterization and enzyme inhibition studies on 5-(2-nitrostyryl)-1,3,4-oxadiazole-2-thiol derivatives. J. Chil. Chem. Soc. 2013;58, (4) :2186.

23. Nafeesa K, Aziz-ur-Rehman, Abbasi M A, Siddiqi S Z, Rasool S, Hussain G, Ahmad I. Synthesis and Biological Screening of S-Substituted Derivatives of 5\{1-[(4-chlorophenyl)sulfonyl]-3-piperidinyl\}-1,3,4oxadiazole-2-yl sulfide. Asian J. Chem. 2015;27(6): 2105-2108,

24. Aziz-Ur-Rehman, Khalid H, Abbasi M A, Gul S, Ahmad I, Arshad S. Synthesis of Potent Antibacterial Agents Derived from5-[1-(Phenylsulfonyl)piperidin-4-yl]-1,3,4oxadiazol-2-thiol. J.Chem.Soc.Pak 2014; 36 (1): 131139

25. Aziz-ur-Rehman, Nafeesa $K$, Abbasi M A, Kashfa $H$, Rasool S, Ahmad I Arshad S. Synthesis, Characterization and Biological Screening of Various Ssubstituted Derivatives of 5-(3-Nitrophenyl)-1,3,4Oxadiazole-2-thiol. Pak. J. Chem. 2013; 3(2): 1-8. 